\title{
Observation of Multivortex Solitons in Photonic Lattices
}

\author{
Bernd Terhalle, ${ }^{1,2}$ Tobias Richter, ${ }^{3}$ Anton S. Desyatnikov, ${ }^{1}$ Dragomir N. Neshev, ${ }^{1}$ Wieslaw Krolikowski, ${ }^{1}$ \\ Friedemann Kaiser, ${ }^{3}$ Cornelia Denz, ${ }^{2}$ and Yuri S. Kivshar ${ }^{1}$ \\ ${ }^{1}$ Nonlinear Physics Center and Laser Physics Center, Research School of Physical Sciences and Engineering, \\ Australian National University, Canberra, ACT 0200, Australia \\ ${ }^{2}$ Institut für Angewandte Physik and Center for Nonlinear Science (CeNoS), Westfälische Wilhelms-Universität, \\ 48149 Münster, Germany \\ ${ }^{3}$ Institut für Angewandte Physik, Technische Universität Darmstadt, Germany \\ (Received 28 February 2008; published 3 July 2008)
}

\begin{abstract}
We report on the first observation of topologically stable spatially localized multivortex solitons generated in optically induced hexagonal photonic lattices. We demonstrate that topological stabilization of such nonlinear localized states can be achieved through self-trapping of truncated two-dimensional Bloch waves and confirm our experimental results by numerical simulations of the beam propagation in weakly deformed lattice potentials in anisotropic photorefractive media.
\end{abstract}

DOI: 10.1103/PhysRevLett.101.013903

PACS numbers: 42.65.Tg, 42.65.Wi, 42.70.Qs

Some of the most spectacular experiments in the field of nonlinear dynamics of coherent light and matter waves in periodic potentials relate to the properties of vortices and vortex flows in optical lattices [1-4]. Dramatic changes of light diffraction or tunneling of matter waves in media with periodically modulated parameters offer novel directions for manipulating waves with a complex phase structure. In optics, self-trapped phase singularities [5] in the form of isolated discrete vortices have been predicted theoretically [6-9] and generated experimentally in square photonic lattices $[1,2,10]$. In the physics of Bose-Einstein condensates, the matter-wave vortices are generated in the form of periodic vortex lattices [11-13].

Multivortex coherent states appear naturally in systems with repulsive interparticle interactions, where they can be confined by external potentials. For attractive interaction, multivortex structures are known to be unstable, and they have been observed only as infinite periodic waves [14]. As a result, spatially localized multivortex states remain unobserved and largely unexplored.

Recently, it was predicted theoretically [15] that photonic lattices with a threefold symmetry can support stable multivortex spatially localized states, in sharp contrast to earlier studied square lattices [16]. Such localized modes originate from a specific type of nonlinear periodic modes, and they became localized in the form of truncated nonlinear Bloch states [17]. Surprisingly, as was shown in extensive numerical simulations, such localized states with high vorticity are stable, whereas their counterparts with lower topological charge experience strong topological instabilities [18]. In this Letter, we report on the experimental observation of topologically stable spatially localized multivortex solitons generated in optically induced hexagonal photonic lattices. We believe that this observation provides the first evidence (in any field of physics) of stable multivortex clusters in systems with attractive nonlinear interaction.
Our experiments are carried out in a 15-mm-long photorefractive crystal of strontium barium niobate (SBN) in which a two-dimensional lattice of a threefold symmetry [see Fig. 1(b)] is induced optically [19] by an interference of three ordinarily polarized plane waves [20]. Because of an intrinsic anisotropy of the photorefractive nonlinear crystal, the induced potential is also strongly anisotropic [18]. To compensate for the asymmetry of the lattice and balance corresponding energy flows between the individual sites, the lattice is deformed from its exact hexagonal
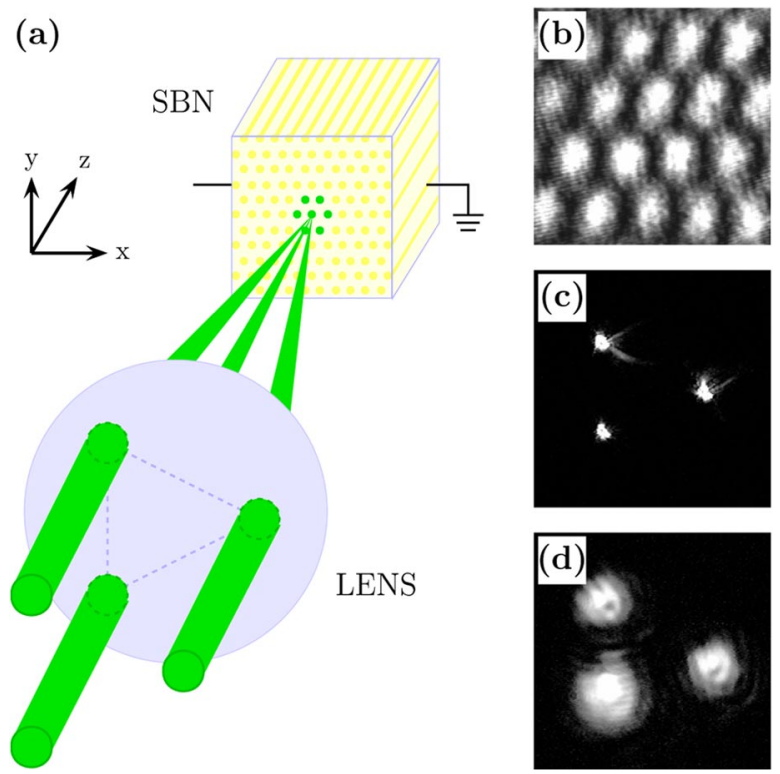

FIG. 1 (color online). Schematic of the experimental setup indicating (a) three extraordinarily polarized beams forming an input probe beam carrying a vortex cluster. (b) Intensity distribution and (c) Fourier image of the lattice field and (d) Fourier image of the probe beam. Note that the hexagonal lattice (b) is stretched with its Fourier components (c) lying at the vertices of the isosceles instead of an equilateral triangle. 
symmetry. More specifically, compared to a truly hexagonal pattern with a lattice constant ratio of $d_{y} / d_{x}=\sqrt{3}$, for the horizontal and vertical direction, respectively, the induced lattice is slightly stretched along the vertical direction having $d_{x}=27 \mu \mathrm{m}$ and $d_{y}=60 \mu \mathrm{m}$. Hence, the ratio of the lattice constants becomes $d_{y} / d_{x} \approx 2.2$. To ensure that the lattice remains stationary along the crystal, all three lattice-forming waves have the same longitudinal $z$ component of their wave vectors. This means that, in the Fourier space $\left(k_{x}, k_{y}\right)$, they are represented by the points located on a ring centered at the origin $k_{x}=k_{y}=0$.

Figure 1(c) shows the Fourier image of the lattice with the three bright spots marking the edges of the first Brillouin zone. To generate a multivortex probe beam, we focus three other extraordinarily polarized beams onto the front face of the crystal [see Fig. 1(a)]. To ensure that the probe beam has the same symmetry as that of the induced lattice, the constituent input beams are adjusted to map the positions of the lattice-forming waves in the Fourier space, as shown in Fig. 1(d). We mention that, in order to achieve localization of the probe beam, its spatial frequency spectrum is kept much broader than that of the lattice waves [see Fig. 1(c)]. In the real space depicted in Fig. 2(a), this arrangement results in an input probe beam having the form of seven distinctive spots forming a hexagonal pattern with the same periods as the lattice and containing six vortices. The vortex positions, indicated by red and blue circles in Fig. 2(b), are visualized experimentally by interfering the probe beam with an inclined broad reference beam.

At low input powers $\approx 20 \mathrm{nW}$, the diffraction of the probe beam leads to a broad output distribution shown in Figs. 3(a) and 3(b). However, at high powers $\approx 1 \mu \mathrm{W}$, the structure becomes localized, and the output intensity distribution features seven well-pronounced spots closely resembling the input.

To show the topological stability of the input structure with a complex phase pattern and to verify experimentally the structure of multivortex solitons, we record the phase
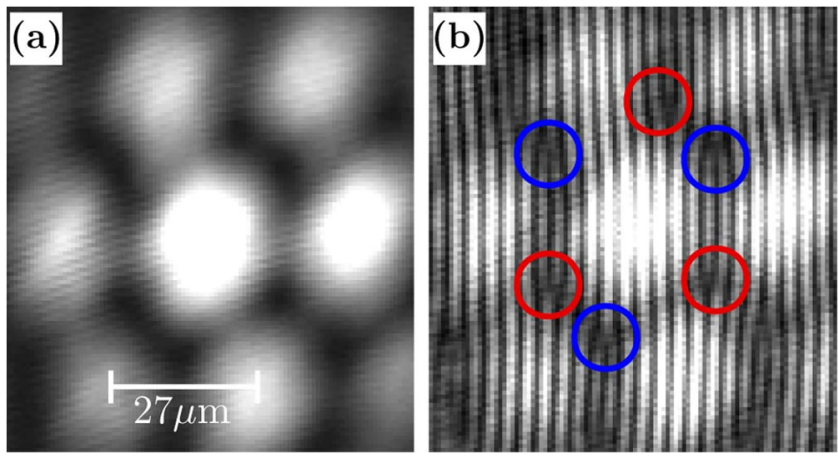

FIG. 2 (color online). (a) Intensity distribution and (b) phase interferogram of the probe input beam. Positions of the vortices are indicated by circles, blue for the topological charge $m=-1$ and red for the topological charge $m=+1$. interferograms of the reference beam and the probe beam at low and high intensities, respectively [see Fig. 4]. It is clearly visible that at low power (in the linear regime) the initial phase profile becomes strongly distorted. While the six initial vortices can still be found in the output field, their positions are changed. In contrast, for high input power of the probe beam (in the nonlinear regime), not only does the beam intensity become self-trapped but also the phase profile retains exactly the same hexagonal vortex pattern of the input beam.

We emphasize that the observation of stable multivortex solitons requires stretching of the photonic lattice. When the lattice is exactly of a hexagonal shape, we still observe self-trapping in the form of seven intensity spots; however, the phase distribution becomes random, and it does not contain a regular cluster of vortices similar to that shown in Fig. 4. Moreover, we find that in this case the output profile experiences strong deformations even for slight perturbations of the input beam. In sharp contrast, the multivortex solitons in the stretched lattice (Figs. 3 and 4) are remarkably robust and are basically insensitive to small deformations of the input beam.

To corroborate our experimental results by numerical simulations, we model the beam propagation through a self-focusing photorefractive crystal using the scalar equation for the slowly varying envelope $A(x, y, z)$ of the probe beam

$$
2 i \frac{\partial A}{\partial z}+\nabla_{\perp}^{2} A-\gamma E_{\mathrm{scr}}\left(I_{\mathrm{tot}}\right) A=0,
$$

where $\nabla_{\perp}^{2} \equiv \partial_{x x}^{2}+\partial_{y y}^{2}$ and $I_{\text {tot }}=\left|A_{\text {latt }}\right|^{2}+|A|^{2}$ is a sum of the intensities of the lattice-forming and probe beams.
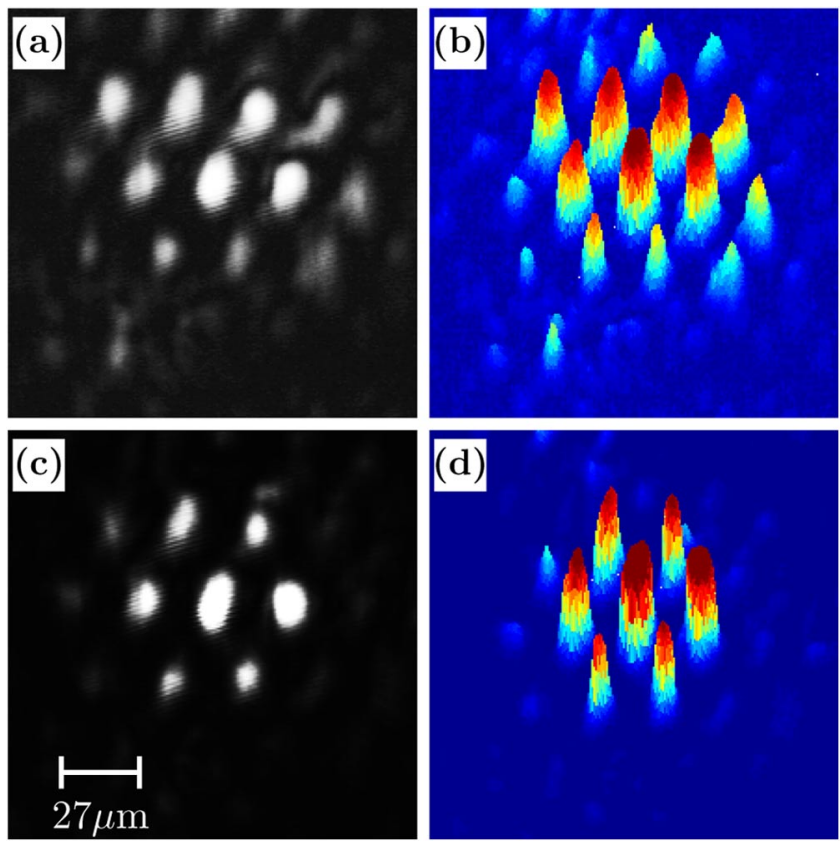

FIG. 3 (color online). Output intensity distributions and corresponding three-dimensional surface plots of the probe beam for (a),(b) low input intensities and (c),(d) high input intensities. 


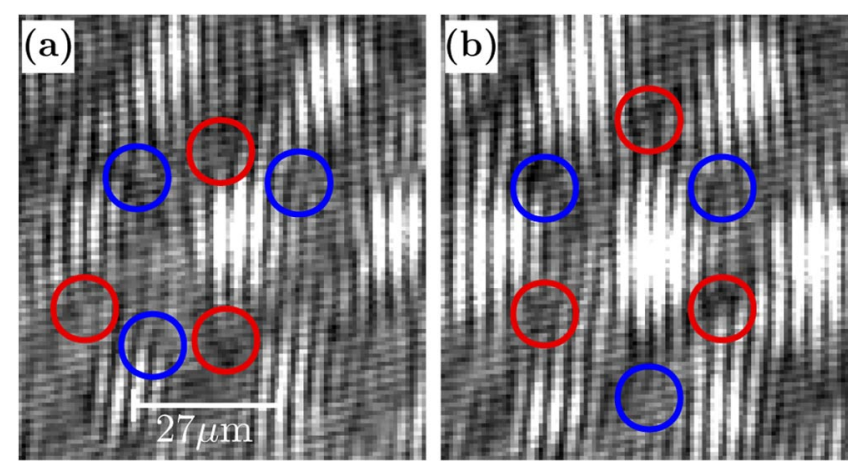

FIG. 4 (color online). Phase interferogram of the probe beam output at (a) low intensity and (b) multivortex soliton at high intensity. Vortex positions are indicated by blue circles for the charge $m=-1$ and by red circles for the charge $m=+1$.

The coefficient $\gamma=k^{2} w_{0}^{2} n_{0}^{2} r_{\text {eff }}$ is proportional to the effective element of the linear electro-optic tensor $r_{\text {eff }}$, and Eq. (1) has been made dimensionless by introducing the transverse $w_{0}$ and longitudinal $z_{0}=k w_{0}^{2}$ length scales, with $k$ and $n_{0}$ denoting the wave vector and refractive index of the unperturbed medium, respectively. Throughout this Letter, we use the values $r_{\text {eff }}=280 \mathrm{pm} / \mathrm{V}, n_{0}=2.35$, and $w_{0}=10 \mu \mathrm{m}$, which yields $z_{0}=2.8 \mathrm{~mm}$. Spatially localized and stationary solutions of Eq. (1) can be found in the form $A(x, y, z)=a(x, y) \exp (i \beta z)$, where $\beta$ is the soliton propagation constant.

The photorefractive nonlinearity is described by the electric screening field $E_{\text {scr }}=-\partial \phi / \partial x$ with the scalar potential $\phi$. Assuming a temporally steady state and neglecting photovoltaic effects, the following expression may be derived for $\phi[21]$ :

$$
\Delta \phi+\nabla \ln \left(1+I_{\text {tot }}\right) \nabla \phi=E_{\text {ext }} \partial_{x} \ln \left(1+I_{\text {tot }}\right),
$$

in which the externally applied homogeneous electric field $E_{\text {ext }}$ is directed along the $x$ axis which coincides with the axis of spontaneous polarization of the crystal ( $c$ axis). The total intensity $I_{\text {tot }}$ is measured in the units of the background illumination, and diffusion effects are neglected. We consider the lattice field in a general form of the superposition of the three interfering plane waves [20]

$$
\begin{aligned}
A_{\text {latt }}= & \exp \left(2 i k_{x} x / 3\right)+\exp \left(-i k_{x} x / 3+i k_{y} y\right) \\
& +\exp \left(-i k_{x} x / 3-i k_{y} y\right) .
\end{aligned}
$$

We start with the intensity profile of an exact hexagonal symmetry when the spatial frequencies $k_{x}$ and $k_{y}$ obey a simple relation $k_{x}=\sqrt{3} k_{y}$. However, the actual symmetry of the induced refractive index lattice is reduced due to the anisotropy of the photorefractive process [22].

In the following, we consider the soliton clusters in the semi-infinite gap [23]; i.e., we assume that all lobes are centered on the lattice sites and only weakly coupled. Then the intensity transfer between the lobes $i$ and $j$ is proportional to $J_{i j}=c_{i j} \sin \left(\phi_{j}-\phi_{i}\right)$, wherein $\phi_{i, j}$ denotes the respective phases and $c_{i j}$ are the coupling coefficients. In order to create a stable vortex cluster, the intensity flows between all lobes should be balanced, i.e., $\sum_{i=1}^{N} J_{i j}=0$ (see details in Ref. [16]). For the structure with $N=7$ lobes and a hexagonal symmetry, this implies that the transverse power flow between each outer lobe and the center lobe has to be twice as high as those between the outer lobes themselves. Under this constraint, the flow condition can be fulfilled only if for all adjacent lobes the coefficients $c_{i j}$ become equal. However, this condition cannot be fulfilled in the case of hexagonal lattices with $k_{x}=\sqrt{3} k_{y}$ due to the photorefractive anisotropy. As a result, at low powers of the input beam, the anisotropic discrete diffraction dominates the dynamics [see Fig. 5(a)]. In the soliton regime shown in Fig. 5(b), the intensity of the probe beam attains a well-defined seven-lobe structure but with an additional modulation resembling that of the diffracted beam. This modulation indicates complex deformations of the power flow [16]. Indeed, the phase map depicted in Fig. 5(c) reveals that only two vortices remain within the localized beam. Hence, in a hexagonal lattice, the vortices are unstable and undergo topological transformations during propagation. Figure 5(d) contains more details of vortex dynamics for the propagation distance much longer than the actual crystal length.

However, the photorefractive anisotropy of the lattice can be compensated for, and, consequently, the energy flow can be balanced by adjusting the ratio $k_{x} / k_{y}$ appropriately. Indeed, a stable phase profile can be achieved for the seven-lobe cluster in this case. This particular profile corresponds to a truncated nonlinear Bloch wave originating from the $M$ point of the lattice spectrum [15].

To determine the necessary deformation (stretching) of the lattice, one needs to evaluate the $c_{i j}$ coefficients for the seven-lobe cluster. To this end, we assign a phase profile with $2 \pi / 3$ jumps between adjacent lobes and calculate the total energy flow between them. Then the balanced flow relations are solved numerically. To find the intensity profiles for both the fundamental solitons and clusters, we use a suitably modified version of the algorithm suggested in Ref. [24] which is able to handle the nonlocal and anisotropic nonlinearity described by Eq. (2).

The results of our numerical simulations with the stretched lattice are summarized in Figs. 5(e)-5(h). The output intensity and phase for the soliton solution are both regular with all six vortices preserved intact. The surface plot in Fig. 5(h) shows weak oscillations of the vortex positions which we ascribe to internal oscillatory modes of the soliton. An important conclusion drawn from these simulations is that, even for propagation distances much longer than the crystal length, these modes do not grow. This indicates stability of the multivortex soliton against small perturbations.

In conclusion, we have generated experimentally topologically stable multivortex solitons in optically induced photonic lattices. We have shown that a weak stretching of the photonic lattice along its vertical direction allows for 

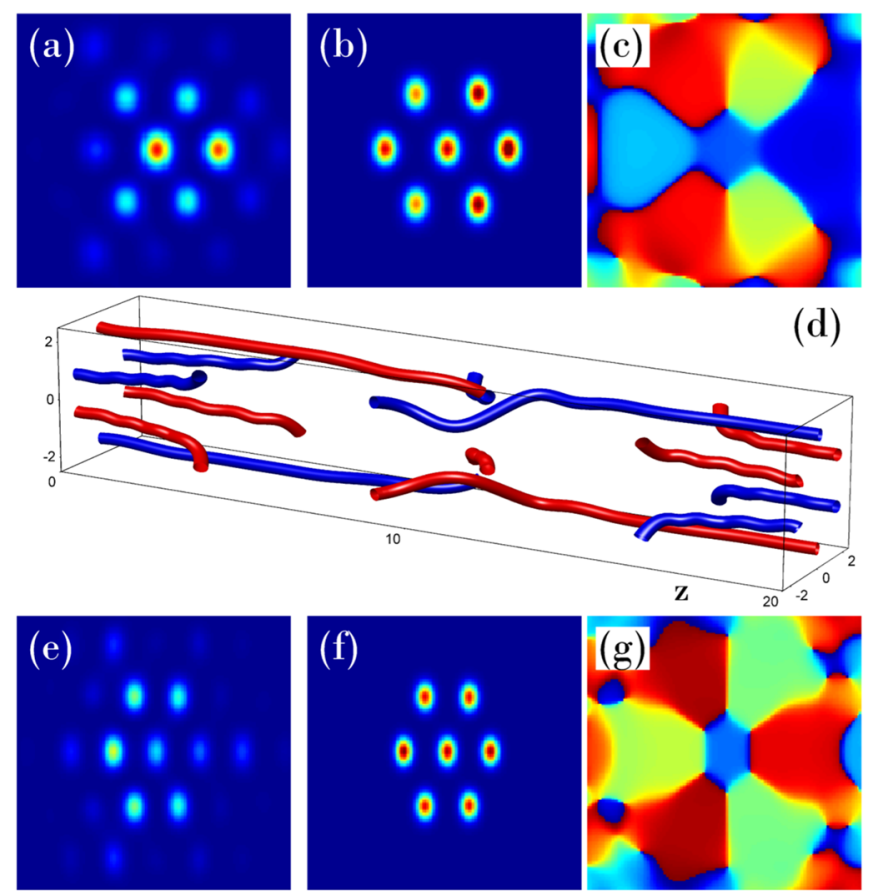

(d)

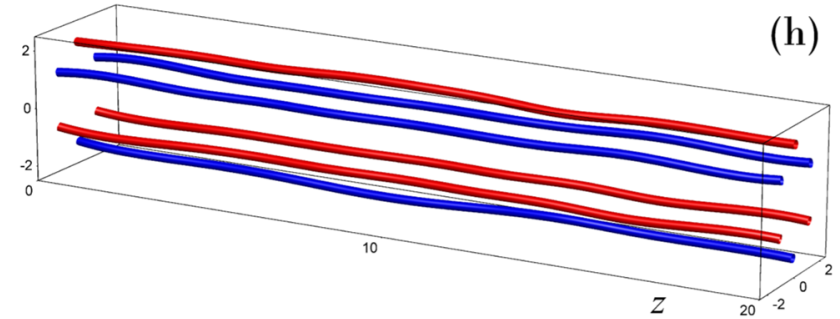

FIG. 5 (color online). Numerical simulations of the propagation of the probe beam in hexagonal lattice (a)-(d) and in the stretched lattice (e)-(h). Diffraction of the probe beam at low intensity is shown in (a) and (e), the self-localization at high intensity in (b) and (f), and phase profiles for the high intensity outputs in (c) and (g). We also show in (d) and (h) the 3D vortex trajectories during propagation; here red curves correspond to vortex charge $m=+1$ and blue curves to charge $m=-1$.

compensating anisotropy of the photorefractive nonlinearity to balance the energy flow and achieve the soliton stability. Our experimental results have been confirmed by extensive numerical simulations of an anisotropic nonlinear model with photorefractive nonlocal response. We believe our demonstrations in nonlinear optics will be useful for the observation of multivortex localized states in other systems, e.g., in Bose-Einstein condensates with attractive interaction in the form of vortex lattices of a finite extent stabilized by two- or three-dimensional periodic potentials.

B. T. thanks the Nonlinear Physics Center and Laser Physics Center at the Australian National University for warm hospitality during his stay in Canberra and acknowledges financial support from the German Academic
Exchange Service (DAAD). This work has been supported by the Australian Research Council through the Discovery Project.

[1] D. N. Neshev, T. J. Alexander, E. A. Ostrovskaya, Yu. S. Kivshar, H. Martin, I. Makasyuk, and Z. Chen, Phys. Rev. Lett. 92, 123903 (2004)

[2] J. W. Fleischer, G. Bartal, O. Cohen, O. Manela, M. Segev, J. Hudock, and D. N. Christodoulides, Phys. Rev. Lett. 92, 123904 (2004).

[3] S. Tung, V. Schweikhard, and E. A. Cornell, Phys. Rev. Lett. 97, 240402 (2006).

[4] V. Schweikhard, S. Tung, and E. A. Cornell, Phys. Rev. Lett. 99, 030401 (2007).

[5] A.S. Desyatnikov, Yu.S. Kivshar, and L. Torner, in Progress in Optics Vol. 47, edited by E. Wolf (Elsevier, Amsterdam, 2005), pp. 291-391.

[6] P. G. Kevrekidis, B. A. Malomed, and Yu. B. Gaididei, Phys. Rev. E 66, 016609 (2002).

[7] J. Yang and Z. H. Musslimani, Opt. Lett. 28, 2094 (2003).

[8] B. B. Baizakov, B.A. Malomed, and M. Salerno, Europhys. Lett. 63, 642 (2003).

[9] J. Yang, New J. Phys. 6, 47 (2004).

[10] G. Bartal, O. Manela, O. Cohen, J. W. Fleischer, and M. Segev, Phys. Rev. Lett. 95, 053904 (2005).

[11] K. W. Madison, F. Chevy, W. Wohlleben, and J. Dalibard, Phys. Rev. Lett. 84, 806 (2000).

[12] V. Schweikhard, I. Coddington, P. Engels, V.P. Mogendorff, and E. A. Cornell, Phys. Rev. Lett. 92, 040404 (2004).

[13] J.R. Abo-Shaeer, C. Raman, J.M. Vogels, and W. Ketterle, Science 292, 476 (2001).

[14] A. S. Desyatnikov, N. Sagemerten, R. Fischer, B. Terhalle, D. Träger, D. N. Neshev, A. Dreischuh, C. Denz, W. Krolikowski, and Yu. S. Kivshar, Opt. Express 14, 2851 (2006).

[15] T. J. Alexander, A.S. Desyatnikov, and Yu. S. Kivshar, Opt. Lett. 32, 1293 (2007).

[16] T. J. Alexander, A. A. Sukhorukov, and Yu. S. Kivshar, Phys. Rev. Lett. 93, 063901 (2004).

[17] T. J. Alexander, E. A. Ostrovskaya, and Yu.S. Kivshar, Phys. Rev. Lett. 96, 040401 (2006).

[18] A. Bezryadina, D. Neshev, A. S. Desyatnikov, J. Young, Z. Chen, and Yu. S. Kivshar, Opt. Express 14, 8317 (2006).

[19] N. K. Efremidis, S. Sears, D. N. Christodoulides, J. W. Fleischer, and M. Segev, Phys. Rev. E 66, 046602 (2002).

[20] C. R. Rosberg, D. N. Neshev, A. A. Sukhorukov, W. Krolikowski, and Yu.S. Kivshar, Opt. Lett. 32, 397 (2007).

[21] A. A. Zozulya and D.Z. Anderson, Phys. Rev. A 51, 1520 (1995).

[22] P. Rose, T. Richter, B. Terhalle, J. Imbrock, F. Kaiser, and C. Denz, Appl. Phys. B 89, 521 (2007).

[23] T. Richter and F. Kaiser, Phys. Rev. A 76, 033818 (2007).

[24] J. J. García-Ripoll and V. M. Pérez-García, SIAM J. Sci. Comput. 23, 1316 (2001). 